\title{
Multiple sclerosis and related challenges to young women's health: Canadian expert review
}

\author{
Galina Vorobeychik*1,2, Denise Black ${ }^{3}$, Paul Cooper ${ }^{4,5}$ \& Ashley Cox ${ }^{6}$ \\ ${ }^{1}$ Department of Medicine, University of British Columbia, Vancouver, BC V5Z 1M9 \\ ${ }^{2}$ Fraser Health Multiple Sclerosis Clinic, Burnaby Hospital, Burnaby, BC V5G 2X6 \\ ${ }^{3}$ Seine River Medical Centre, Winnipeg, MB R2N OA5 \\ ${ }^{4}$ Department of Clinical Neurological Sciences, Western University, London, ON N6A 3K7 \\ ${ }^{5}$ Schulich School of Medicine \& Dentistry, London, ON N6A 5C1 \\ ${ }^{6}$ Department of Urology, Dalhousie University, Halifax, NS B3H 1 Y 6 \\ *Author for correspondence: Tel.: +604 412 6405; Galina.Vorobeychik@fraserhealth.ca
}

\section{Practice points}

- A total of $60 \%$ of Canadian adults diagnosed with multiple sclerosis (MS) are between the ages of 20 and 49 years, and twice as many young women as men have MS.

- The most common comorbidities among Canadian patients with MS were found to be depression, hypertension, migraine, hypercholesterolemia and anxiety.

- Depression and anxiety are associated with progression of disability in MS, and comorbid depression can also lead to reduced adherence to disease-modifying therapy.

- Migraines occur up to three-times more frequently in patients with MS than among those in the general population, and are more common in women than in men.

- Pregnancy represents an immunotolerant state for women with MS and has no effect on the course of disease; however, the incidence of complicated delivery was increased and the postpartum period is associated with an increased risk of relapse.

- Urinary and/or bowel issues are experienced by up to $90 \%$ of patients with MS at some point in the course of their disease, and nearly one-half of patients report that these issues are serious enough to interrupt their daily lives at least some of the time.

- Management of the female patient with MS should employ a multidisciplinary approach following the five C's: comprehensive, coordinated, community based, care of the MS patient and close to home.

Multiple sclerosis (MS) is among the most common chronic neurological diseases, with a highly variable degree of disability during its long-term course. The majority of patients develop significant permanent disability later in life. MS is often diagnosed in women of childbearing age, with a 3:1 ratio of young women to young men with MS. Comorbidities such as depression, anxiety, migraines and reproductive, urological and bowel issues are common and negatively impact patients' quality of life. The objective of this supplement is to review the most common comorbidities occurring in young women with MS, and to propose a multidisciplinary, holistic approach to management.

First draft submitted: 25 February 2020; Accepted for publication: 15 April 2020; Published online: 6 May 2020

Keywords: anxiety $\bullet$ depression $\bullet$ migraine $\bullet$ multiple sclerosis $\bullet$ reproductive health $\bullet$ urological and bowel conditions

Multiple sclerosis (MS) is among the most common chronic neurological diseases diagnosed in young adults [1]. MS is a complex condition, during which different mechanisms can be involved. It is an inflammatory disease of the central nervous system and may include not only immune reactions but also a multitude of degenerative processes [2]. Immune cells become activated in the periphery, cross the blood-brain barrier and attack the protective myelin covering the nerves of the brain and spinal cord [3,4]. While slight damage to the myelin results in minor interruptions of nerve impulses, more substantial damage or recurrent injuries of the myelin may result in complete disruption of nerve impulses and damage to the nerves themselves. In addition, normally appearing white matter 
Table 1. Estimated prevalence of multiple sclerosis across Canada.

\begin{tabular}{|ll|}
\hline Province or region & Prevalence of multiple sclerosis (cases per 100,000 population) \\
\hline Quebec & 180 \\
\hline Ontario & 230 \\
\hline British Columbia & 240 \\
\hline Prairie provinces & 340 \\
\hline Atlantic provinces & 350 \\
\hline Data taken from [10]. & \\
\hline
\end{tabular}

is not absolutely free of MS-related changes, as has been shown with advanced imaging techniques [5]. These factors contribute to different degrees of disability, heterogeneous clinical manifestation and highly variable and unpredictable progression [6]. MS places a considerable burden on patients and their families, as well as on the healthcare system and society as a whole. Although the cause of MS remains unknown, it is believed that genetics, possible exposure to infections and unidentified environmental factors play a role [3].

The objective of this supplement is to review certain MS-related comorbidities in young women and propose a multidisciplinary holistic approach to managing these patients. The comorbidities discussed include depression, anxiety, migraines and reproductive, urological and bowel issues.

\section{Epidemiology of MS in Canadian women}

An estimated 2.3 million people live with MS worldwide [1]. In North America, the prevalence is 140 cases per 100,000 population [1]; approximately $70 \%$ of people with MS are women. Among adults 20-40 years of age, the ratio of women to men with MS has increased from 2:1 to 3:1 in the last 30 years [7]. An estimated 93,500 Canadians were reported as diagnosed with MS based on the most recent data available from the 2010/2011 Neurological Conditions Prevalence File and the 2011 Survey on Living with Neurological Conditions [8]. The overall prevalence of MS in Canada is 260 cases per 100,000 population, with $60 \%$ of adults diagnosed with MS between the ages of 20 and 49 years of age [9]. Sex-specific prevalence rates in Canada are 418 and 159 cases per 100,000 population for women and men, respectively. There are also regional differences in the prevalence of MS across Canada (Table 1) [10]. It is predicted that in 2031, the number of Canadians with MS will increase to 133,635 , with a prevalence of 620 cases per 100,000 women and 220 cases per 100,000 men [11].

MS confers a significant economic burden on society as it usually affects young adults during their prime careerand family-building years. In Canada, the total per capita healthcare cost, excluding out-of-pocket expenses, for adults aged 20 years and older with MS was approximately $\$ 16,800$ in 2011 . Total annual health sector costs for MS are expected to reach approximately $\$ 2.0$ billion by 2031 after accounting for additional expenditures due to MS and excluding out-of-pocket expenses [11].

\section{Treatment initiation}

The clinical course of MS is highly variable, ranging from patients with relatively mild disease even years after diagnosis to those with an early aggressive course and rapid accumulation of disability. Before initiating MS therapy, clinicians must consider the nature of the disease and long-term treatment plan, which is likely to be influenced by patient- and disease-related characteristics [6]. Early MS treatment initiation is associated with a better clinical outcome [12].

\section{MS-related comorbidities}

A systematic review of the incidence and prevalence of comorbidities in MS revealed significant gaps in the epidemiological knowledge relating to comorbidities in MS worldwide [13]. From 1905 to 2012, 52.2\% of studies were conducted in Europe, 33.7\% in North America and only 9.6\% in Asia, 2.8\% in Australia/New Zealand and 1.6\% in South America. The five most prevalent comorbidities were depression, anxiety, hypertension, hyperlipidemia and chronic lung disease. Little high quality information is available regarding the incidence or prevalence of comorbidities in MS at any given point in the disease course. An analysis of 949 patients from four Canadian MS clinics revealed the most common comorbidities among patients with MS to be depression (29.0\%), hypertension (17.8\%), migraine (17.3\%), hypercholesterolemia (12.4\%) and anxiety (11.5\%) [14]. 
Comorbidities have a significant impact on patients with MS. Not only do comorbidities affect disability progression, quality of life (QoL), need for healthcare and mortality risk [8,15], but they can also cause diagnostic delay, exacerbate risk of hospitalization and increase the risk of relapse [16]. In addition, comorbidities play a role in the choice of disease-modifying therapy (DMT), tolerance to therapy and long-term adherence [17]. In addition, comorbid diseases significantly increase societal costs to the management of chronic, progressive illnesses such as MS [18,19]. MS-related comorbidities that have a particular impact on young women with MS include depression and anxiety [14], migraines [20], reproductive issues [7] and urological and bowel issues [21].

\section{Depression \& anxiety in young women with MS}

A systematic review and meta-analysis of 58 published studies with a total of 87,756 patients with MS reported a pooled mean prevalence of $30.5 \%$ for depression and $22.1 \%$ for anxiety [22]. The presence of clinically significant depressive or anxiety symptoms was higher than the prevalence of true disorders (depression: 35 vs $21 \% ; \mathrm{p}=0.001$; anxiety: 34 vs $10 \% ; \mathrm{p}<0.001)$. Subgroup analysis by region showed a significant difference $(\mathrm{p}=0.028)$ in the prevalence of depression (11.3\% in Europe, 27.3\% in North America and 28.1\% for Australia, the Middle East and Asia together); however, a similar subgroup analysis for anxiety did not show any regional difference in prevalence. In Canada, $29.0 \%$ of patients with MS have comorbid depression and $11.5 \%$ have comorbid anxiety [14].

It is believed that the same biological processes involved in the immunopathogenesis of MS are responsible for mediating depression. Increases in proinflammatory cytokines, activation of the hypothalamic-pituitary-adrenal axis and reduction in neurotrophic factors observed in patients with MS may be responsible for the increased rate of depression seen in MS [23,24]. If the biological processes involved in MS contribute to the pathogenesis of depression and if depression negatively affects the neurophysiological processes related to immune function, it follows that treatment of depression may positively affect MS disease progression [24].

Depression and anxiety contribute to the challenges in treating patients with MS. Depression has a negative effect on QoL and is second only to disability status in affecting health-related QoL (HRQoL) in MS [14]. Furthermore, the presence of depression or anxiety is associated with disability progression in MS [25]. Depression can also be a factor in decreased adherence to DMTs in MS [26,27]. Evidence shows that patients with MS and comorbid depression have significantly lower adherence to DMT compared with patients with MS only ( $p<0.01$ ), and adherence to antidepressant therapy for at least 6 months is associated with improved adherence to DMT therapy [26].

Depression and anxiety in MS may be partly due to stress. MS is a major stressor, not only for the patient, but also for the patient's family. A combination of factors may contribute to stress, which can manifest as severe and acute or as a chronic condition. Stressors may be physical (e.g., significant disability caused by MS), psychological (e.g., dependence on family, friends and caregivers to carry out activities of daily living), social (e.g., withdrawal from social activities) or financial (e.g., inability to continue working) [28-30]. The uncertainty and unpredictability of the disease course can also contribute to major stress.

A systematic review and meta-analysis of interventions for depression and anxiety in patients with MS showed that the severity of depression improved in nine trials using psychological interventions and in three trials using pharmacological interventions [31]. Due to a lack of data, the analysis was unable to determine the effectiveness of interventions for anxiety. Cognitive behavioral therapy (CBT) is effective in improving depression and mental health in patients with MS [31,32]. CBT in the various studies among MS patients could be done individually or as a group, in-person, online or by telephone, and typically involved standard procedures of behavioral activation and cognitive restructuring used with patients who have depression. The antidepressants paroxetine, sertraline and desipramine have been studied in randomized, controlled trials in patients with MS [33]. Sertraline has shown efficacy in reducing depression as measured with the Beck Depression Inventory ${ }^{\circledR}\left(\mathrm{BDI}^{\circledR}\right)$, while desipramine had positive effects on depression according to the Hamilton Depression Rating Scale. Paroxetine, in contrast, did not show a statistically significant improvement in depression compared with placebo. Duloxetine, fluvoxamine, moclobemide and sertraline have been studied in open-label trials in patients with MS; all four demonstrated efficacy in improving depression [33].

Since depression and anxiety may be difficult to identify in the clinical setting, validated screening tools should be considered to identify patients who could benefit from treatments for depression or anxiety. Tools that assess not only depression and anxiety directly but also fatigue and cognitive decline may be useful. According to guidelines for the assessment of psychiatric disorders in individuals with MS published by the American Academy of Neurology, the BDI and a two-question screen are possibly effective and may be considered for identifying individuals with depression [34]. In both the original and revised BDI, patients rank according to their severity (0-3) a series of 
self-evaluative statements for each of 21 categories of behaviors and attitudes that describe a specific behavioral manifestation of depression [35,36]; e.g., for evaluation of sadness, the patient chooses between 0 ('I do not feel sad'), 1 ('I feel sad'), 2 ('I am sad all the time and I can't snap out of it') and 3 ('I am so sad and unhappy that I can't stand it'). The two-question screen includes one question each about depressed mood ("During the past month, have you often been bothered by feeling down, depressed or hopeless?") and diminished interest or pleasure ("During the past month, have you often been bothered by little interest of pleasure in doing things?'); an affirmative response to either question produces a positive screen [37].

Obtaining information from the patient directly may be sufficient to identify depression or anxiety, or the clinician may decide to seek collateral information separately from the patient's caregiver who may be in a better position to provide evidence for depression or anxiety. Finally, obtaining longitudinal data over time may show changes in performance on questionnaires that may indicate the onset or worsening of depression or anxiety.

\section{Migraines in young women with MS}

Migraines occur two- to three-times more frequently in patients with MS than in the general population [38-40], with studies reporting prevalence rates between 21 and 69\% [39,40]. Women with MS are more likely to experience migraines and headaches than men with MS [41]. The association between MS and migraine is unclear; current hypotheses suggest that migraine may precede the development of MS, that migraines and MS share a common pathophysiology, and that migraines experienced by patients with MS represent a distinct subtype [40]. It should be noted that MS itself does not cause migraines, as migraines are by definition a primary headache disorder and cannot be caused by other conditions [42].

MS can, however, cause migraine-like headaches. Several hypotheses have been put forward to explain the higher incidence of migraine-like headaches in individuals with MS. One hypothesis proposes that the presence of B-cell follicles in the meninges and gyri results in the transfer of antibodies, leading to an inflammatory process. This meningeal inflammatory process is believed to cause meningeal irritation, which, in turn, leads to migrainelike headaches [41,43]. Merkler et al. suggested that migraine-like headaches are caused by inflammation-mediated demyelination of the cortex, which leads to accelerated cortical spreading depression (CSD); CSD is a hallmark for migraine [44]. Gee et al. proposed that the presence of active MS plaques in the periaqueductal gray matter of the midbrain causes migraine-like headaches. This hypothesis is based on findings that MS plaques in the midbrain correlate with a higher likelihood of developing migraine-like headaches [45].

Stress, depression and anxiety are believed to contribute to migraines and headaches in individuals with MS. A study conducted in patients with MS and chronic headaches who responded to a standardized questionnaire identified stress as the most common trigger of headache $(78 \% ; \mathrm{p}<0.001)$ [46]. The presence of depression, anxiety, and migraine in individuals with MS leads to loss of productivity, increased financial and social burden on the individual and society and decreased HRQoL $[20,47]$.

The recommended treatment approach for migraines in patients with MS is the same as for the general population and includes both pharmacological and nonpharmacological modalities [20]. Pharmacological treatment includes treatment for acute attacks, as well as daily preventive medications that decrease the frequency of migraines. Agents approved by Health Canada for the acute management of migraine attacks include triptans, select nonsteroidal anti-inflammatory drugs (NSAIDs; acetylsalicylic acid, ibuprofen, naproxen and diclofenac rapid dissolve), acetaminophen and dihydroergotamine mesylate; other NSAIDs, neuroleptics and antiemetics are commonly employed as off-label therapies [20,48-50]. Approved prophylactic agents include topiramate, propranolol (betaadrenergic blocker), flunarizine (calcium-entry blocker) and pizotifen hydrogen malate (serotonin and tryptamine antagonist), while common off-label migraine prophylaxis is given in the form of antiepileptic drugs, antidepressants and angiotensin-converting enzyme inhibitors [20,51]. Injection of onabotulinumtoxinA into selected cranial muscles is also approved in Canada as a prophylactic agent against migraine [20,52]. Three members of a new class of monoclonal antibodies that inhibit calcitonin gene-related peptide (CGRP) - erenumab, galcanezumab, and fremanezumab - are available in Canada for antimigraine prophylaxis. The safety, efficacy and tolerability of these agents for the prophylactic management of episodic and chronic migraine were established in Phase III trials (Phase II for erenumab in chronic migraine) [53-55]. Nonpharmacological approaches such as CBT and relaxation therapy aim to decrease the frequency and severity of migraines that interfere with normal daily functioning at home and at work [20].

In Canada, unmet needs for young women with MS who suffer from migraines or migraine-like headaches include difficulty in obtaining an accurate diagnosis due to the dearth of headache neurologists as well as long-waiting lists 
to see headache specialists. In addition, there is a lack of familiarity with the guidelines regarding the treatment of individuals with migraines or migraine-like headaches. Furthermore, there are barriers to nonpharmacological treatment modalities in Canada: many of these modalities are not covered by insurance plans, patients may be unwilling or unable to bear the associated cost, many of these modalities require coaching and support and patients may be overwhelmed by the added responsibility of maintaining these treatments. Finally, there is no single pharmacological therapy that is effective for all patients with migraines or migraine-like headaches. These unmet needs contribute to challenges to the health of young women with MS.

\section{Reproductive health issues in young women with MS}

At puberty, women begin to have an elevated risk for MS [56]. It is believed that hormonal changes that occur at puberty directly influence immune pathways toward proinflammatory activity. Thus, the physiological interaction between the reproductive and immune systems may explain the increased risk for MS that begins at the time of menarche. In developed countries, there has been a trend toward earlier menarche, which is a risk factor for both MS and for earlier onset of MS symptoms. Menstruation is associated with a proinflammatory state, and women with MS may experience 'mini relapses' around the time of menstruation. The etiology of these 'mini relapses' is unknown; however, it is likely that the proinflammatory nature of menstruation along with the precipitous decline in both estrogen and progesterone are contributing factors [56,57]. In contrast, pregnancy is generally a time of immunotolerance.

MS is unlikely to affect female fertility [56]. Women with MS may be evaluated for infertility after 6 months of unsuccessful attempts, compared with 12 months for women without MS. Sexual dysfunction, which is fivetimes more common among women with MS than in the general population, may play a role in infertility. A large proportion of women with MS experience sexual dysfunction at some point. Issues related to sexual dysfunction include both physical (urinary urgency, urinary leakage, pain during intercourse and reduced mobility) and psychosocial (decreased libido, interpersonal difficulties, depression and body image issues). Women with MS who opt for assisted reproductive technology are at increased risk of disease relapse, especially with unsuccessful cycles [56]. The use of gonadotropin-releasing hormone agonists may increase this relapse risk [56]. Regarding fertility planning, DMTs should ideally be discontinued prior to conception; however, this is a complex issue and depends on factors such as disease activity and the specific DMT. Health Canada recommends use of interferon-beta (IFN- $\beta$ ) or glatiramer acetate (GA) during pregnancy only when the potential benefits of treatment outweigh the possible hazards in the physician's estimation [58,59]. Similarly, continuation of natalizumab is acceptable only if the agent is clearly needed [60]. Fingolimod has recently been contraindicated in women who are pregnant or in women of childbearing potential who are not using effective contraception due to its association with an elevated risk of major congenital malformations [61]. Health Canada recommends that fingolimod be discontinued at least 2 months before planning pregnancy. Coyle et al. (2019) recommended that natalizumab be avoided in the third trimester because of its high risk of fetal thrombocytopenia and hemolytic anemia [62].

Current guidelines developed by the World Health Organization and the American College of Obstetricians and Gynecologists recommend that women with MS have access to all methods of contraception [63,64]. These include long-acting reversible contraceptive methods, such as intrauterine devices and systems (IUDs and IUSs; available in Canada) and implantable rods, as well as oral contraceptives. An IUS is an IUD that contains a levonorgestrel (LNG) secreting core cylinder. The LNG is slowly released inside the uterus. The contraceptive effect is through local mechanisms. IUSs do not always inhibit ovulation, and monthly fluctuations in hormones continue to occur [62]. However, menstruation may or may not be suppressed depending on the device and patient. The benefit of IUSs is their high degree of contraceptive efficacy that is independent of patient compliance. Oral contraceptives do not interact with DMTs [56], are unlikely to worsen the course of MS, and may actually provide the benefit of stabilizing hormone levels throughout the treatment cycle. This effect may be extended by skipping the hormone-free interval (i.e., continuous use), which may prevent declines in estrogen and progesterone and thereby inhibit menstruation.

The obstetrical management of pregnancy in women with MS is similar to the general obstetrical population $[56,62]$. Women with MS should have vitamin D levels monitored and supplemented as necessary and should take folic acid daily [56,62]. Women with MS should be followed closely for urinary tract infections (UTIs), to which they are more susceptible than women without MS [65]. In general, pregnancy is an immunotolerant state with fewer relapses, especially in the third trimester (70\% fewer relapses) [66]. Most importantly, pregnancy does not alter the course of MS [66]. High-dose corticosteroids may be used for relapses during pregnancy, but dexamethasone and betamethasone should be avoided because they are known to cross the placenta [67]. Women with MS have slightly 
higher rates of induced labor. Women with increasing disability have higher incidences of Cesarean section and operative vaginal delivery; however, it is unclear whether this is primarily due to patient or physician preference [56]. One retrospective analysis of administrative database claims showed elevated risks for Cesarean section (4-16\% increase), infection (18-22\% increase) and preterm delivery (20-30\% increase) [68]. Another analysis of administrative database claims found that significantly more women with MS submitted claims related to premature labor, infection and acquired fetal damage than women without MS [69].

The postpartum period is generally recognized as a time for increased risk of relapse, especially the first postpartum trimester. Women with very active MS, those who relapse during pregnancy and those with no prior DMT use are at particularly high risk of postpartum relapse and may consider rapid initiation or reinitiation of a DMT [62]. High-dose steroids may also be used for acute exacerbations. In the postpartum period, women with MS should pay particular attention to sleep, pelvic floor issues, signs of UTI and signs and symptoms of postpartum depression [56].

Currently, data are inconclusive regarding whether breastfeeding is beneficial in diminishing disease activity and reducing the risk of relapse. There are limited data on IFN- $\beta$ use in breastfeeding patients; a study involving six nursing mothers found that subclinical levels of the drug (estimated relative infant dose $0.006 \%$ of the maternal dose) are excreted in breast milk [70]. Health Canada recommends that the physician and patient decide between IFN- $\beta$ and breastfeeding [58]. In the absence of clinical data for GA in nursing women, Health Canada advocates caution for its administration in a nursing woman [59].

\section{Urological \& bowel issues in young women with MS}

Up to $90 \%$ of patients with MS experience urinary and/or bowel issues at some point during the course of disease, and nearly $50 \%$ report that bowel issues are serious enough to interrupt their work, social activities, and other aspects of daily life at least some of the time [21]. Urological and bowel issues are key contributors to disease burden in women with MS, and the increased severity of urological and bowel issues contribute to poorer quality of life [71,72]. A survey of 9341 patients with MS (7720 [77.4\%] were females) revealed that $66.2 \%$ of respondents reported being mildly, moderately or severely bothered by bladder and bowel symptoms [71]. Among respondents, $62.1 \%$ reported that their healthcare providers inquired about bladder symptoms and $51.1 \%$ of patients' healthcare providers inquired about bowel symptoms. In addition, 5.7\% of respondents reported the change in QoL as much better, $17.3 \%$ as better and $20.3 \%$ as a little better following treatment for bladder, bowel and sexual symptoms.

Urological issues in women with MS include urinary incontinence, hesitancy, retention, urgency and incomplete bladder emptying [73,74]. Urinary symptoms can be classified using the mnemonic FUN WISE into storage symptoms (frequency, urgency and nocturia) and voiding symptoms (weak stream, intermittent stream, straining to void and emptying). Overactive bladder is the most common urinary dysfunction in patients with MS. It is a storage dysfunction characterized by the sudden and compelling desire to urinate that is difficult to defer and by frequent daytime or nighttime urination $[21,75]$. Patients with MS are roughly twice as likely to have bothersome urgency and frequency of urination compared with the general population [75,76]. A further $25 \%$ of patients with MS report urinary retention (inability to empty the bladder) with elevated post-void residual urine volume [77]. This voiding dysfunction is due to a weak bladder muscle (detrusor) or lack of coordination between the sphincter and the bladder, which may cause obstruction (also referred to as detrusor sphincter dyssynergia). Complications of urinary symptoms have an impact on patients with MS, including decreased QoL, increased healthcare costs, increased hospital admissions and increased morbidity due to incontinence, skin breakdown, UTIs, kidney stones, renal deterioration and fistulas $[21,75]$.

The work-up to identify urinary symptoms may include taking a patient's history, physical examination, urine studies, voiding diary, uroflowmetry/scan, renal ultrasound, cystoscopy and urodynamic studies [78]. The treatment of urological issues depends on the symptoms as well as findings of investigations and should be individualized to the patient. Treatments include conservative therapies and medications (Box 1), as well as self-catheterization, onabotulinumtoxinA, neuromodulation and surgery [74,75,79-81].

Bowel dysfunction affects approximately 39-73\% of patients with MS. Evidence shows the presence of bowel symptoms correlates with disease progression as well as disease duration [82]. Approximately $50 \%$ of patients with MS experience constipation and around $20 \%$ have ongoing issues with fecal incontinence. The impact of bowel disorders on QoL is significant, contributing to the inability to work or even leave the house, and thus leading to depression [82]. In addition, bowel disorders are associated with multiple debilitating disorders, including hemorrhoids, abdominal pain, bloating, fecal impactions, nausea and autonomic dysreflexia. 
Box 1. Conservative therapies and medications used to treat urinary dysfunction in patients with multiple sclerosis.

\section{Conservative therapies}

Fluid restriction

- $25 \%$ reduction in fluid can lead to improvement in urinary symptoms

- Limit caffeine to $100 \mathrm{mg} /$ day

- Avoid carbonated beverages and artificial sweeteners

Smoking cessation

- Smoking may be related to worsening overactive bladder

Bladder retraining

- Timed voiding schedule, "urge suppression techniques"

- Frequent opportunities at assisted voiding

- Clothing that is quick to remove for ease of voiding

- Commodes at the bedside, urinals

Pelvic floor muscle training

- Requires a specialized physiotherapist

- Long-term therapy (9 weeks) has been shown to benefit MS patients

Medications

Anticholinergics (e.g., solifenacin, fesoterodine and oxybutynin)

- Reduce bladder contractions, improve volume voided, and decrease incontinence episodes

- Adverse effects include dry mouth, constipation, blurry vision, dry eyes and difficulty voiding (increased postvoid residual urine volume)

$\beta 3$-agonists (e.g., mirabegron)

- Reduce bladder contractions, improve volume voided, and decrease incontinence episodes

- Adverse effects may include hypertension

Data taken from $[74,75,79]$.

MS: Multiple sclerosis.

According to the Rome IV criteria, constipation is defined by the presence of $\geq 2$ of the following symptoms in at least $25 \%$ of defecations [82]:

- Straining during defecation;

- Hard or lumpy stools;

- Sensation of incomplete evacuation;

- Sensation of anorectal blockage or obstruction;

- Use of manual maneuvers to facilitate evacuation (includes digitation to the perineum);

- $\leq 3$ defecations per week;

- Rare occurrence of loose stools without the use of laxatives;

The causes of constipation and fecal incontinence in patients with MS are multifactorial, as shown in Boxes 2 and 3.

Investigations for bowel disorders include taking a family history, physical examination, a 2-week bowel diary and anorectal physiological studies such as colonic transit time studies, endoanal ultrasound, magnetic resonance imaging and computed tomography [21]. Dietary and lifestyle changes are the first line of treatment for preventing and managing bowel disturbances. These include fiber, fluid and fitness. When dietary and lifestyle changes are insufficient to resolve bowel issues, other options include stool softeners, laxatives, suppositories, enemas, prescription medications (eg., lubiprostone and linaclotide), manual stimulation, pelvic floor therapy and sacral nerve stimulation [21].

\section{Holistic approach to managing MS in young women}

The multiple common comorbidities associated with MS in young women - depression, anxiety, migraines, and reproductive, urological and bowel issues - all confer a significant impact on a patient's QoL. QoL in patients with MS is a practical concern related not just to satisfaction or happiness but to their functioning and productivity in 
Box 2. The multifactorial origin of constipation in patients with multiple sclerosis.

- Poor diet

- Prolonged colonic transit

- Loss of supraspinal modulation

- Drugs

- Anorectal hyposensitivity

- Abdominal wall weakness forces

- Pelvic floor dyssynergia

- Incoordination

- Alterations of rectoanal inhibitory reflux

- Rectal hyposensitivity

- Altered rectal compliance

- Behavioural factors

- Difficulty accessing the toilet

- Previous episodes of fecal incontinence

Data taken from [82].

Box 3. The multifactorial origin of fecal incontinence in patients with multiple sclerosis.

- Uncontrolled colonic peristalsis

- Anorectal hyposensitivity

- Anal sphincter weakness

- Prolonged rectoanal inhibitory reflux

- Altered rectal compliance

- Loss of voluntary control of defecation (frontal lobe)

This table was created using data from [82].

society. Therefore, identifying and managing these comorbidities plays an important role in treating this population of MS sufferers [29,30].

Because MS-related symptoms are complex and interrelated, optimal management requires a holistic, multidisciplinary approach, including patient education and empowerment [83,84]. In addition to delaying disease progression and managing symptoms, assessing and promoting QoL and general well-being should be a principal goal of treatment. For many young women with MS, this remains an unmet need [30].

Ideally, young women diagnosed with MS should be evaluated in MS specialty centers with multidisciplinary teams who are able to identify patients' individual needs and to design effective treatment programs aimed at minimizing disease progression and maximizing QoL. Although DMTs may reduce symptoms and relapses and delay progression, many have little impact on QoL. Improvement in the patient's overall well-being may be achieved with other treatments aimed at improving MS-related symptoms and managing comorbidities [30].

The holistic approach to treating patients with MS should encompass the five C's:

- Comprehensive;

- Coordinated;

- Community based;

- Care of MS patient;

- Close to home.

These objectives can be best met through a holistic-care strategy involving a multidisciplinary team (Table 2) [85]. This holistic approach should offer clinical care, patient education, and the opportunity for research into improved care for patients with MS. The clinical care team consists of the neurology team and the symptom management team. The role of the neurology team is to establish the diagnosis of MS, manage MS with DMTs, and provide care during relapses. The neurology team comprises neurologists and nurses, who are supported by specialists in neuro-ophthalmology, radiology, electrophysiology and laboratory. The symptom management team is divided into three groups, each with specific responsibilities for managing a unique set of symptoms: cognitive and mood 


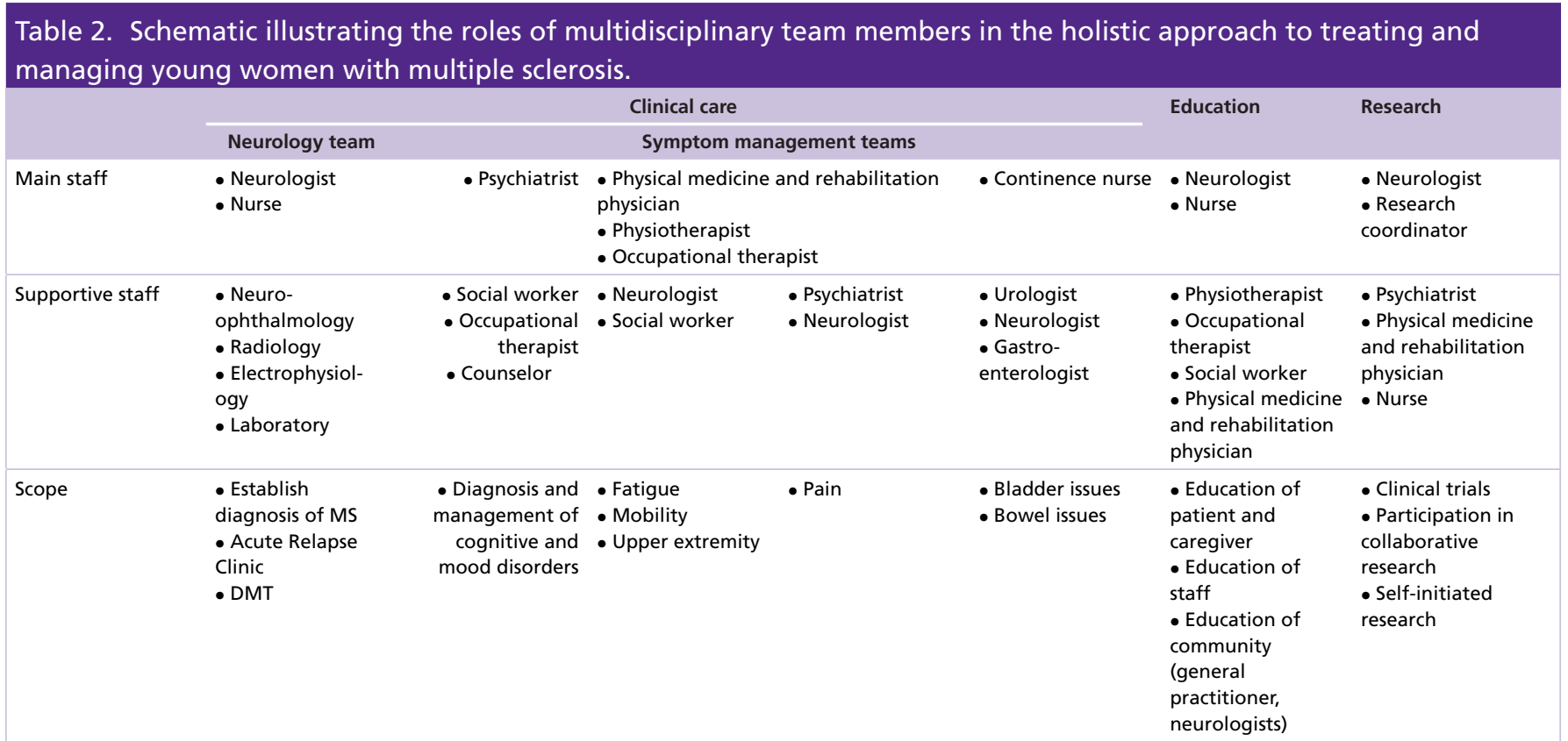

Used with permission from Dr Vorobeychik [85].

DMT: Disease-modifying therapy.

disorders; fatigue, mobility, upper extremity issues and pain; and bladder and bowel issues (Table 2). The first group is led by a psychiatrist and supported by a social worker, an occupational therapist and a counselor, frequently in the setting of a mental health unit close to the patient's home. The second group is led by a physical medicine and rehabilitation physician, a physiotherapist and an occupational therapist, and is supported by a neurologist and a social worker (responsible for managing fatigue, mobility and upper extremity issues) as well as a psychiatrist and neurologist (responsible for managing pain). This group frequently involves staff from the local health authority unit, which can provide patient assessment in the home setting. The third group is led by a continence nurse, and is supported by a urologist, a neurologist and a gastroenterologist.

The multidisciplinary holistic approach also offers education for the patient, the patient's family, the caregiver as well as the staff and community of the MS specialty center (Table 2). This is particularly important for newly diagnosed patients who may have questions about topics such as prognosis, disease progression and disability. Education also offers the opportunity for the patient's family to benefit from counseling. The main staff members involved in education consist of a neurologist and a nurse, with support from a physiotherapist, an occupational therapist, a social worker and a physical medicine and rehabilitation physician.

Finally, the multidisciplinary holistic approach offers the opportunity for research into the treatment and management of MS, whether through clinical trials, participation in collaborative research or self-initiated research (Table 2). Research would be led mainly by a neurologist and one or more research coordinators, supported by a psychiatrist, a physical medicine and rehabilitation physician and a nurse.

The multidisciplinary holistic approach is helpful for the patient, caregivers and healthcare personnel. Patients and caregivers benefit through better MS management complemented by comprehensive education and support. Clinicians are able to perform more accurate risk stratification before and during the disease, better predict response to medications, assess the risk of disease progression, determine disease prognosis and manage non-neurological comorbidities.

\section{Future perspective}

The incorporation of a precision medicine approach, which identifies biomarkers for multiple sclerosis and other diseases, and personalized medicine with patient-focused strategy will add value to the holistic care of MS patients $[86,87]$. The multidisciplinary, holistic approach represents a promising evolution in the model of care for young women with MS. It can be extrapolated and used in other chronic diseases, which require complex, lifelong collaboration of healthcare professionals to provide patients with full support and help them to maintain their QoL. 


\section{Conclusion}

MS primarily afflicts young women, who are also particularly at risk for many of the most common MS comorbidities. Conditions such as depression, anxiety, migraines and urinary and bowel issues adversely affect progression of disability, risk of relapse, QoL, healthcare requirement and risk of mortality. Young women with MS are optimally cared for with a holistic multidisciplinary approach, which also benefits the healthcare team.

Financial \& competing interests disclosure

The author(s) declared the following potential conflicts of interest with respect to the research, authorship, and/or publication of this article.

$\mathrm{G}$ Vorobeychik reports that she has received research support and/or presenter honorarium from Berlex, Biogen, Celgene, Genzyme, Roche, Sanofi, Serono, Novartis, Teva Neuroscience. D Black has nothing to disclose in association with this work. P Cooper reports that he has given talks for Allergan, Teva, Novartis and Aralez on the subject of headache and received honoraria. He has also participated in advisory boards for Novartis, Lilly and Teva. He has no stock in any of the companies or other commercial interest. A Cox reports that she has received honoraria as a speaker from Astellas and Pfizer.

The authors have no other relevant affiliations or financial involvement with any organization or entity with a financial interest in or financial conflict with the subject matter or materials discussed in the manuscript apart from those disclosed. The authors have no other relevant affiliations or financial involvement with any organization or entity with a financial interest in or financial conflict with the subject matter or materials discussed in the manuscript apart from those disclosed.

The authors thank A Styhler and J Alexander, SNELL Medical Communication, for editorial assistance with the preparation of this paper. Editorial assistance was funded by Teva Canada.

Open access

This work is licensed under the Attribution-NonCommercial-NoDerivatives 4.0 Unported License. To view a copy of this license, visit http://creativecommons.org/licenses/by-nc-nd/4.0/

\section{References}

Papers of special note have been highlighted as: $\bullet$ of interest; $\bullet \bullet$ of considerable interest

1. Multiple Sclerosis International Federation (MSIF). Atlas of MS 2013: Mapping Multiple Sclerosis Around the World. www.msif.org/wp-content/uploads/2014/09/Atlas-of-MS.pdf

2. Correale J, Marrodan M, Ysrraelit MC. Mechanisms of neurodegeneration and axonal dysfunction in progressive multiple sclerosis. Biomedicines 7(1), 14 (2019).

3. Evans C, Beland SG, Kulaga S et al. Incidence and prevalence of multiple sclerosis in the Americas: a systematic review. Neuroepidemiology 40(3), 195-210 (2013).

4. Multiple Sclerosis Society of Canada. What is MS?. (2019). https://mssociety.ca/about-ms/what-is-ms

5. Moll NM, Rietsch AM, Thomas S et al. Multiple sclerosis normal-appearing white matter: pathology-imaging correlations. Ann. Neurol. 70(5), 764-773 (2011).

6. Freedman MS, Selchen D, Prat A, Giacomini PS. Managing multiple sclerosis: treatment initiation, modification, and sequencing. Can. J. Neurol. Sci. 45(5), 489-503 (2018).

7. Mendibe Bilbao M, Boyero Durán S, Bárcena Llona J et al. Multiple sclerosis: pregnancy and women’s health issues. Neurologia 34(4), 259-269 (2019).

8. Gilmour H, Ramage-Morin PL, Wong SL. Multiple sclerosis: prevalence and impact. Statistics Canada, Catalogue no. 82-003-X Health Reports, 29(1), 3-8 (2018).

9. Widdifield J, Ivers NM, Young J et al. Development and validation of an administrative data algorithm to estimate the disease burden and epidemiology of multiple sclerosis in Ontario, Canada. Mult. Scler. 21(8), 1045-1054 (2015).

- Provides an important approach to assess burden of disease.

10. Beck CA, Metz LM, Svenson LW et al. Regional variation of multiple sclerosis prevalence in Canada. Mult. Scler. 11(5), 516-519 (2005).

11. Amankwah N, Marrie RA, Bance JC et al. Multiple sclerosis in Canada 2011 to 2031: results of a microsimulation modelling study of epidemiological and economic impacts. Health. Promot. Chronic. Dis. Prev. Can. 37(2), 37-48 (2017).

-. This model is important for planning the future impact of Multiple sclerosis (MS) in the Canadian population.

12. Kavaliunas A, Manouchehrinia A, Stawiarz L et al. Importance of early treatment initiation in the clinical course of multiple sclerosis. Mult. Scler. 23(9), 1233-1240 (2017).

13. Marrie RA, Cohen J, Stuve $\mathrm{O}$ et al. A systematic review of the incidence and prevalence of comorbidity in multiple sclerosis: overview. Mult. Scler. 21(3), 263-281 (2015). 
14. Berrigan LI, Fisk JD, Patten SB et al. Health-related quality of life in multiple sclerosis: direct and indirect effects of comorbidity. Neurology 86(15), 1417-1424 (2016).

-. This paper is a comprehensive review of Canadian data in MS and comorbidities.

15. Overs S, Hughes CM, Haselkorn JK et al. Modifiable comorbidities and disability in multiple sclerosis. Curr. Neurol. Neurosci. Rep. 12(5), 610-617 (2012).

16. Kowalec K, McKay KA, Patten SB et al. Comorbidity increases the risk of relapse in multiple sclerosis: a prospective study. Neurology 89(24), 2455-2461 (2017).

17. Pardo G, Jones DE. The sequence of disease-modifying therapies in relapsing multiple sclerosis: safety and immunologic considerations. J. Neurol. 264(12), 2351-2374 (2017).

18. Marrie RA, Horwitz RI. Emerging effects of comorbidities on multiple sclerosis. Lancet Neurol. 9(8), 820-828 (2010).

19. Moss BP, Rensel MR, Hersh CM. Wellness and the role of comorbidities in multiple sclerosis. Neurotherapeutics 14(4), $999-1017$ (2017).

20. Husain F, Pardo G, Rabadi M. Headache and its management in patients with multiple sclerosis. Curr. Treat. Options. Neurol. 20(4), 10 (2018).

21. Multiple Sclerosis Society of Canada. Managing bladder \& bowel issues in MS. (2015). https://mssociety.ca/uploads/files/2085naep-2015-book-english.pdf

22. Boeschoten RE, Braamse AMJ, Beekman ATF et al. Prevalence of depression and anxiety in multiple sclerosis: a systematic review and meta-analysis. J. Neurol. Sci. 372, 331-341 (2017).

- In this comprehensive analysis, the authors demonstrated the difficulties of research of depression and anxiety within the field of MS.

23. Gold SM, Irwin MR. Depression and immunity: inflammation and depressive symptoms in multiple sclerosis. Immunol. Allergy. Clin. North. Am. 29(2), 309-320 (2009).

24. Pucak ML, Carroll KA, Kerr DA et al. Neuropsychiatric manifestations of depression in multiple sclerosis: neuroinflammatory, neuroendocrine, and neurotrophic mechanisms in the pathogenesis of immune-mediated depression. Dialogues. Clin. Neurosci. 9(2), 125-139 (2007).

25. McKay KA, Tremlett H, Fisk JD et al. Psychiatric comorbidity is associated with disability progression in multiple sclerosis. Neurology 90(15), e1316-e1323 (2018).

26. Tarrants M, Oleen-Burkey M, Castelli-Haley J et al. The impact of comorbid depression on adherence to therapy for multiple sclerosis. Mult. Scler. Int. 2011, 271321 (2018).

27. Treadaway K, Cutter G, Salter A et al. Factors that influence adherence with disease-modifying therapy in MS. J. Neurol. 256(4), 568-576 (2009).

28. Landfeldt E, Castelo-Branco A, Svedbom A et al. The long-term impact of multiple sclerosis on the risk of divorce. Mult. Scler. Relat. Disord. 24, 145-150 (2018).

29. National Multiple Sclerosis Society. Rehabilitation: recommendations for persons with multiple sclerosis. (2004). www.nationalmssociety.org/NationalMSSociety/media/MSNationalFiles/Brochures/Opinion-Paper-Rehabilitation-Recommendations-f or-Persons-with-MS.pdf

30. Zwibel HL, Smrtka J. Improving quality of life in multiple sclerosis: an unmet need. Am. J. Manag. Care. 17(Suppl. 5), S139-S145 (2011).

31. Fiest KM, Walker JR, Bernstein CN et al. Systematic review and meta-analysis of interventions for depression and anxiety in persons with multiple sclerosis. Mult. Scler. Relat. Disord. 5, 12-26 (2016).

32. Simpson R, Simpson S, Ramparsad N et al. Mindfulness-based interventions for mental well-being among people with multiple sclerosis: a systematic review and meta-analysis of randomised controlled trials. J. Neurol. Neurosurg. Psychiatry 90(9), 1051-1058 (2019).

33. Nathoo N, Mackie A. Treating depression in multiple sclerosis with antidepressants: a brief review of clinical trials and exploration of clinical symptoms to guide treatment decisions. Mult. Scler. Relat. Disord. 18, 177-180 (2017).

- Provides a valuable review of antidepressant treatments in an MS population.

34. Minden SL, Feinstein A, Kalb RC et al. Evidence-based guideline: assessment and management of psychiatric disorders in individuals with MS: report of the Guideline Development Subcommittee of the American Academy of Neurology. Neurology 82(2), 174-181 (2014).

35. Beck AT, Ward CH, Mendelson M, Mock J, Erbaugh J. An inventory for measuring depression. Arch. Gen. Psychiatry 4, 561-571 (1961).

36. Beck AT, Steer RA, Brown GK. Manual for the Beck Depression Inventory-II. The Psychological Corporation, TX, USA (1996).

37. Whooley MA, Avins AL, Miranda J, Browner WS. Case-finding instruments for depression. Two questions are as good as many. J. Gen. Intern. Med. 12(7), 439-445 (1997).

38. Applebee A. The clinical overlap of multiple sclerosis and headache. Headache 52(Suppl.2), 111-116 (2012).

39. Kister I, Caminero AB, Monteith TS et al. Migraine is comorbid with multiple sclerosis and associated with a more symptomatic MS course. J. Headache Pain 11, 417-425 (2010). 
40. Peckel L. Migraine and multiple sclerosis: connection or coincidence? Neurology Advisors. 1-4, (2018). www.neurologyadvisor.com/adv isor-channels/headache-migraine-advisor/migraine-and-multiple-sclerosis-connection-or-coincidence

41. Mohrke J, Kropp P, Zettl UK. Headaches in multiple sclerosis might imply an inflammatorial process. PLoS ONE 8(8), e69570 (2013).

42. World Health Organization (WHO). Headache Disorder. (2016). www.who.int/news-room/fact-sheets/detail/headache-disorders

43. Magliozzi R, Howell O, Reeves $\mathrm{C}$ et al. A gradient of neuronal loss and meningeal inflammation in multiple sclerosis. Ann. Neurol. 68(4), 477-493 (2010).

44. Merkler D, Klinker F, Jurgens T et al. Propagation of spreading depression inversely correlates with cortical myelin content. Ann. Neurol. 66(3), 355-365 (2009).

45. Gee J, Chang J, Dublin A et al. The association of brainstem lesions with migraine-like headache: an imaging study of multiple sclerosis. Headache 45(6), 670-677 (2005).

46. Tabby D, Majeed MH, Youngman B et al. Headache in multiple sclerosis: features and implications for disease management. Int. J. MS. Care. 15(2), 73-80 (2013).

- This paper is a comprehensive review of the frequent comorbidity of headache in MS.

47. Marrie RA, Horwitz R, Cutter et al. Cumulative impact of comorbidity on quality of life in MS. Acta Neurol. Scand. 125(3), 180-186 (2012).

48. Charles A. Migraine. N. Engl. J. Med. 377(6), 553-561 (2017).

49. Colman I, Friedman B, Brown M et al. Parenteral dexamethasone for acute severe migraine headache: meta-analysis of randomized controlled trials for preventing recurrence. BMJ. 336(7657), 1359-1361 (2008).

50. Rizzoli P. Acute and preventive treatment of migraine. Continuum 18(4), 764-782 (2012).

51. Silberstein S. Preventive migraine treatment. Continuum 21(4), 973-989 (2015).

52. Barbanti P, Ferroni P. Onabotulinum toxin a in the treatment of chronic migraine: patient selection and special considerations. J. Pain Res. 10, 2319-2329 (2017).

53. Jain S, Yuan H, Spare N et al. Erenumab in the treatment of migraine. Pain Manag. 8(6), 415-426 (2018).

54. Förderreuther S, Zhang Q, Stauffer VL, Aurora SK, Láinez MJA. Preventive effects of galcanezumab in adult patients with episodic or chronic migraine are persistent: data from the Phase III, randomized, double-blind, placebo-controlled EVOLVE-1, EVOLVE-2, and REGAIN studies. J. Headache Pain 19(1), 121 (2018).

55. Silberstein SD, Dodick DW, Bigal ME et al. Fremanezumab for the preventive treatment of chronic migraine. N. Engl. J. Med. 377(22), 2113-2122 (2017).

56. Rankin K, Bove R. Caring for women with multiple sclerosis across the lifespan. Curr. Neurol. Neurosci. Rep. 18(7), 36 (2018).

- This review looks at the progression and management of the female MS patient from her childbearing years through menopause.

57. Maybin JA, Critchley HO. Menstrual physiology: implications for endometrial pathology and beyond. Hum. Reprod. Update 21(6), 748-761 (2015).

58. Biogen Canada Inc. AVONEX ${ }^{\circledR}$ (interferon beta-1a) Product Monograph.

59. Teva Canada Ltd. COPAXONE ${ }^{\circledR}$ (Glatiramer acetate injection) Product Monograph.

60. Biogen Canada Inc. TYSABRI ${ }^{\circledR}$ (natalizumab) Product Monograph.

61. Health Canada. Healthcare professional letter: GILENYA ${ }^{\circledR}$ (fingolimod) - risk of congenital malformations.

62. Coyle PK, Oh J, Magyari M et al. Management strategies for female patients of reproductive potential with multiple sclerosis: an evidence-based review. Mult. Scler. Relat. Disord. 32, 54-63 (2019).

63. World Health Organization (WHO). Medical Eligibility Vriteria for Contraceptive Use. (2015). www.who.int/reproductivehealth/ publications/family_planning/MEC-5/en

64. American College of Obstetricians and Gynecologists (ACOG). Committee Opinion No. 642: Increasing Access to Contraceptive Implants and Intrauterine Devices to Reduce Unintended Pregnancy. Obstet. Gynecol. 126(4), e44-e48 (2015).

65. Phe V, Pakzad M, Curtis C et al. Urinary tract infections in multiple sclerosis. Mult. Scler. 22(7), 855-861 (2016).

66. Airas L, Kaaja R. Pregnancy and multiple sclerosis. Obstet. Med. 5(3), 94-97 (2012).

67. Siroos B, Harirchian MH. Multiple sclerosis and pregnancy: what a neurologist may be asked for? Iran. J. Neurol. 13(2), 57-63 (2014).

68. MacDonald SC, McElrath TF, Hernández-Díaz S. Pregnancy outcomes in women with multiple sclerosis. Am. J. Epidemiol. 188(1), 57-66 (2019).

69. Houtchens MK, Edwards NC, Schneider G et al. Pregnancy rates and outcomes in women with and without MS in the United States. Neurology 91(17), e1559-e1569 (2018).

70. Hale TW, Siddiqui AA, Baker TE. Transfer of interferon $\beta$-1a into human breastmilk. Breastfeed Med. 7(2), 123-125 (2012).

71. Wang G, Marrie RA, Fox RJ et al. Treatment satisfaction and bothersome bladder, bowel, sexual symptoms in multiple sclerosis. Mult. Scler. Relat. Disord. 20, 16-21 (2018). 
-. This complete review provides important Canadian data on a key subject of patient care.

72. Lee KS, Choo MS, Seo JT et al. Impact of overactive bladder on quality of life and resource use: results from Korean Burden of Incontinence Study (KOBIS). Health Qual. Life Outcomes 13, 89 (2015).

73. Rodrigues de Almeida CR, Carneiro K, Fiorelli R et al. Urinary dysfunction in women with multiple sclerosis: analysis of 61 patients from Rio de Janeiro, Brazil. Neurol. Int. 5(4), e23 (2013).

74. Multiple Sclerosis Society of Canada. Bladder Dysfunction in MS. (2012). https://mssociety.ca/en/pdf/bladder-dysfunction.pdf

75. Khalaf KM, Coyne KS, Globe DR et al. Lower urinary tract symptom prevalence and management among patients with multiple sclerosis. Int. J. MS Care 17(1), 14-25 (2015).

76. Coyne KS, Sexton CC, Thompson CL et al. The prevalence of lower urinary tract symptoms (LUTS) in the USA, the UK and Sweden: results from the Epidemiology of LUTS (EpiLUTS) study. BJU. Int. 104(3), 352-360 (2009).

77. Sammarco AG, Orasanu B, Mahajan ST. The bladder in MS: a review. J. Neurol. Neurophysiol. 5(3), 1-7 (2014).

78. Aharony SM, Lam O, Corcos J. Evaluation of lower urinary tract symptoms in multiple sclerosis patients: review of the literature and current guidelines. Can. Urol. Assoc. J. 11(1-2), 61-64 (2017).

79. National Multiple Sclerosis Society. Bladder Problems. (2019). www.nationalmssociety.org/Symptoms-Diagnosis/MS-Symptoms/ Bladder-Dysfunction\#section-4

80. Jhang JF, Kuo HC. Novel applications of onabotulinumtoxinA in lower urinary tract dysfunction. Toxins 10(7), (2018).

81. Gaziev G, Topazio L, Iacovelli V et al. Percutaneous tibial nerve stimulation (PTNS) efficacy in the treatment of lower urinary tract dysfunctions: a systematic review. BMC Urol. 13, 61 (2013).

82. Preziosi G, Gordon-Dixon A, Emmanuel A. Neurogenic bowel dysfunction in patients with multiple sclerosis: prevalence, impact, and management strategies. Degener. Neurol. Neuromuscul. Dis. 8, 79-90 (2018).

83. Embrey N. Multiple sclerosis: managing a complex neurological disease. Nurs. Stand. 29(11), 49-58 (2014).

84. Shah P. Symptomatic management in multiple sclerosis. Ann. Indian. Acad. Neurol. 18(Suppl. 1), S35-S42 (2015).

85. Vorobeychik G, Beauregard N, Savedia-Cayabyab S et al. Developing a new multiple sclerosis clinic model in Canada. J. Neurol. Sci. 238(Suppl. 1), S245 (2005).

86. Comabella M, Sastre-Garriga J, Montalban X. Precision medicine in multiple sclerosis biomarkers for diagnosis, prognosis, and treatment response. Curr. Opin. Neurol. 29(3), 254-262 (2016).

87. Giovannoni G. Personalized medicine in multiple sclerosis. Neurodegener. Dis. Manag. 7(Suppl. 6), 13-17 (2017). 
